\title{
Dual Blockade of the Renin-Angiotensin System: A Strategy that Should Be Reconsidered in Cardiorenal Diseases?
}

\author{
Mei Mei ${ }^{a}$ Zulian Zhou ${ }^{b}$ Qian Zhang ${ }^{c}$ YiChen $^{d}$ Hongwen Zhao ${ }^{c}$ \\ Bingbing Shen ${ }^{c, e}$
}

aDepartment of Nephrology, The People's Hospital of Shapingba District, Chongqing, China; ${ }^{b}$ Department of Nephrology, Qianjiang Central Hospital, Chongqing, China; 'Department of Nephrology, The First Hospital Affiliated to Army Medical University, Chongqing, China; ${ }^{\mathrm{d} C o l l e g e ~ o f ~ P h a r m a c e u t i c a l ~ S c i e n c e s ~ \& ~ C h i n e s e ~ M e d i c i n e, ~ S o u t h w e s t ~}$ University, Chongqing, China; 'Department of Nephrology, Chongqing University Central Hospital, Chongqing Emergency Medical Center, Chongqing, China

\author{
Keywords \\ Dual blockade strategy · Renin-angiotensin system . \\ Cardiorenal diseases
}

\begin{abstract}
Studies on pharmacological mechanisms demonstrated that a strategy of dual renin-angiotensin system (RAS) blockade may have a synergistic effect in the treatment of cardiorenal diseases and may reduce adverse reactions. However, some previous clinical studies reported that dual RAS blockade did not significantly benefit many patients with cardiorenal diseases and increased the risk of hyperkalemia, hypotension and renal function damage. Therefore, the current clinical guidelines suggest that the combined use of angiotensin-converting enzyme inhibitors (ACEls) and angiotensin receptor blockers (ARBs) should be used with caution in the clinic. However, these studies enrolled older patients with cardiovascular risk factors, and the results of these trials may not be generalized to the overall population. Some clinical evidence suggests that the combination of low-dose ACEls and ARBs leads to more effective RAS blockade with few adverse effects. The advent of new RAS inhibitors with superior pharmacological effects provides a more suitable
\end{abstract}

drug choice for individualized therapy for dual RAS blockade. Therefore, the choice of appropriate ARBs/ACEls for individualized therapy based on patient condition may be a better way to improve the efficiency and safety of the dual RAS blockade strategy.

(c) 2021 S. Karger AG, Basel

\section{Introduction}

The renin-angiotensin system (RAS) has been among the most important therapeutic targets in the field of cardiorenal vasculature since it was proposed in the 1990s. RAS inhibitors (RASis), ranging from angiotensin-converting enzyme inhibitors (ACEIs) and angiotensin receptor blockers (ARBs) to renin inhibitors, are continuously developed. RASis are widely used in the clinic, and each drug has unique characteristics that play a very important role in the treatment of cardiorenal diseases. However, the combined use of RASis in the clinic is con-

Second address for correspondence: Department of Nephrology, Chongqing University Central Hospital, Chongqing Emergency Medical Center, No. 1 Jiankang Street, Chongqing 400014 (China).

$\begin{aligned} & \text { karger@karger.com } \\ & \text { www.karger.com/nef }\end{aligned}$
Karger ${ }^{\prime /}$


troversial because the pharmacological mechanisms may achieve synergistic effects, but clinical observations are contradictory. The benefit of dual RAS blockade in the field of cardiorenal diseases was first documented in the CALM study, which found that combination therapy reduced albuminuria more effectively than candesartan or lisinopril monotherapy in patients with hypertension, type 2 diabetes, and microalbuminuria [1]. Therefore, some scholars proposed that the combined use of RASis would improve treatment efficacy in patients [2]. Because reduced proteinuria is correlated with reduced renal and cardiovascular events, the antiproteinuric effect of the dual RAS blockade strategy was expected to achieve longterm renoprotection and cardioprotection. Nephrologists are more supportive of this combination therapy than cardiovascular doctors [3]. However, due to the results of some clinical randomized controlled trials (RCTs) on the possible risks of combined blockade regimens, the European Drug Administration issued a clear warning for the combined use of ARBs and ACEIs in 2014 [4]. This report highlights the results of the following clinical randomized studies: ONTARGET, ALTITUDE, and VANEPHRON-D. The results of these studies suggest that dual RAS blockade regimens increase the incidence of adverse events, including hyperkalemia, hypotension, and renal failure, in patients with cardiovascular risk factors [5-7]. Therefore, ACEIs and ARBs are not recommended in current clinical guidelines. However, some clinical studies showed that dual RAS blockade may significantly benefit some specific populations [8]. The advent of new RASis with superior pharmacological effects provides a more suitable drug choice for individualized therapy for dual RAS blockade. Based on this background, the present article primarily reviews some important research results published in this field in recent years and analyzes and evaluates the safety and clinical value of the dual RAS blockade strategy.

\section{Regulation and Balance of the Renin-Angiotensin System}

The components of the RAS primarily include renin, angiotensin peptides, angiotensin-converting enzyme (ACE), and receptors (Fig. 1) [9]. AngII is especially important because it is the strongest pressor substance currently known [10]. However, ACE2 and angiotensin 1-7 (Ang1-7) also play important roles in maintaining the balance of the RAS [11-13]. ACE2 is a monopeptidase that cleaves an amino acid from the carboxyl end of a substrate. The substrates of ACE2 include AngI and AngII. ACE2 directly changes AngII into Ang1-7, which has vasodilating effects, and it degrades AngI into Ang1-9, which further forms Ang1-7 via other enzymes in vivo. Similar to bradykinin, Ang1-7 is a vasodilator that maintains balance in the RAS. Therefore, the activity of ACE2 plays an important role in regulating the balance of the RAS. Scientists are developing new therapies that are in preclinical and early clinical stages, including recombinant ACE2 protein [14]. However, not all ACEIs have the ability to modulate ACE2. Early drugs, such as captopril and enalapril, have almost no regulatory effect on ACE2, but subsequent drugs, such as perindopril, upregulate ACE2, which strengthens its capacity to control blood pressure and protect the heart, kidney, and other target organs via the ACE2/Ang1-7/MAS axis. Notably, some ACEIs and ARBs (e.g., olmesartan, losartan, and azilsartan) regulate the activity of ACE2 and promote the production of Ang 1-7 and bradykinin [15]. These new RASIs with superior pharmacological effects should be given priority in the dual RAS blocking strategy.

ACEIs and ARBs have similar side effects, namely hypotension, hyperkalemia, renal function deterioration, and fetal malformations [16]. Because these 3 drugs act on different parts of the RAS, they should theoretically have synergistic effects. For example, ACEIs inhibit ACE activity, reduce the production of AngII, aldosterone, and vasopressin, and increase renin secretion, but their blockade efficiency does not reach $100 \%$. Some early marketed drugs did not modulate AngII or aldosterone. In contrast, ARBs block the binding of AngII to the angiotensin 1 receptor (AT1R), which directly blocks the post-receptor effect of AT1 and partially activates the AT2 receptor. Because AngII cannot bind AT1R or be rapidly decomposed, the concentration of AngII in the body increases. As previously mentioned, newly listed ACEIs, such as perindopril, upregulate ACE2 and convert AngII into Ang1-7 [11]. Therefore, the combination of ACEIs and ARBs make good pharmacological sense because ARBs directly block AT1R, and ACEIs convert elevated AngII into the vasodilator Ang1-7. The combination of ACEIs with ARBs had a greater effect than monotherapy on blood pressure and left ventricular hypertrophy in spontaneously hypertensive rats [17]. Thangaraju et al. [18] demonstrated that the combination of ACEI (ramipril) and ARB (telmisartan) showed improvement in the histopathological and biochemical changes in the kidneys of diabetic rat model. These animal experiments support the hypothesis that ACEIs with ARBs are possibly synergistic, 


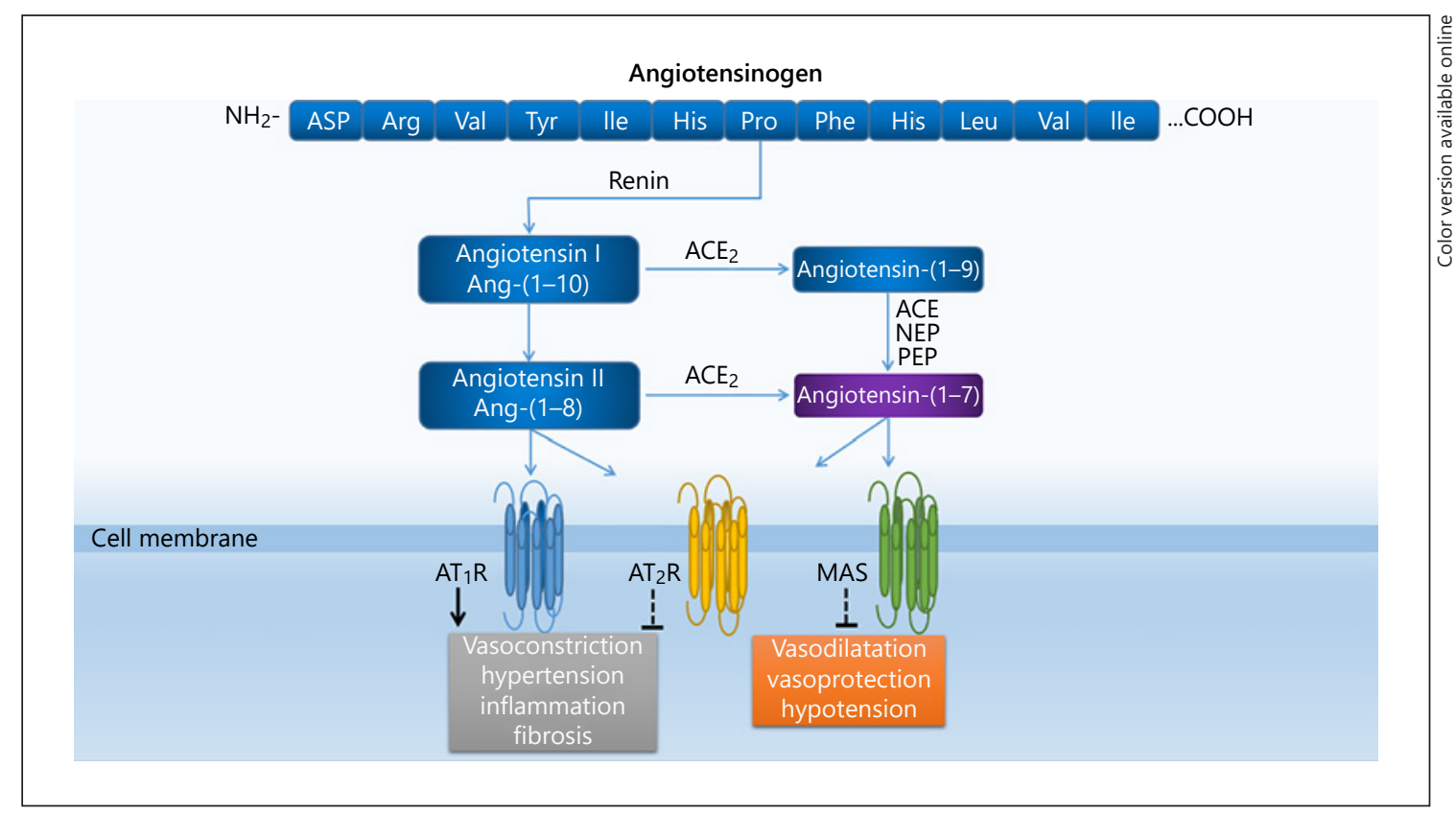

Fig. 1. ANG peptides and receptors in the RAS signaling pathway. Renin cleaves angiotensinogen into Ang I. Ang I can also be cleaved by ACE to Ang II, which stimulates AT1R and AT2R. Ang I can be cleaved into Ang(1-9) by ACE2, and Ang(1-9) can further diverge into Ang(1-7) via ACE/PEP/NEP. Ang II may further di- verge via ACE2 into Ang(1-7), which binds to the AT2R and MAS receptor. RAS, renin-angiotensin system; ACE, angiotensin-converting enzyme; ACEIs, angiotensin-converting enzyme inhibitors; ANG, angiotensin; Ang I, angiotensin I; Ang II, angiotensin II; AT1R, angiotensin 1 receptor; AT2R, angiotensin 2 receptor. which is the basis for a concept of "dual blockade strategy." Cherney et al. [19] found that a combination of renin inhibitors and ACEIs had a good synergistic effect on endothelial function, arteriosclerosis, and renal function in type 1 diabetes [19]. Although the dual blockade is theoretically feasible, the clinical observations are controversial.

\section{The Efficacy and Safety of Dual Renin-Angiotensin System Blockade Strategy}

As mentioned above, RASis with different targets in the RAS are the basis of combination therapy. Because of the promise of the dual blockade effect on RAS, scholars initially investigated the effects of combining ACEIs and ARBs. Some studies, such as CALM and COOPERATE, showed positive effects of the combination for reducing proteinuria [1, 20]. The Valsartan Heart Failure Trial (Val-HeFT) and CHARM-added studies showed that combination therapy was beneficial for patients with chronic heart failure, and it reduced the readmission rate of patients with heart failure $[21,22]$. However, there are too few similar studies, the sample size collected in this study was small, its persuasive power is limited, and the COOPERATE study was withdrawn because of an issue with informed consent. Several subsequent clinical trials, including the ONTARGET, ALTITUDE, and VA NEPHRON-D studies [5-7], found that combination therapy did not significantly increase the clinical benefits for the cardiac and renal endpoint events but increased the risk of hyperkalemia, hypotension, and acute renal injury. Therefore, the current cardiovascular guidelines, including the 2017 American Heart Association (AHA) guidelines and the 2018 European Society of Cardiology (ESC) guidelines [23, 24], do not recommend the combination of ACEIs and ARBs. Other studies clearly showed that dual blockade of RAS was not recommended, and the optimal dose of a single inhibitor remained the preferred strategy $[25,26]$. A review of the details of the classic ONTARGET study, which compared the efficacy of ramipril and telmisartan in high-risk cardiovascular patients (nearly 10,000 high-risk diabetic patients with terminal organ damage), showed that the combination therapy increased the risk of renal endpoint events, hypotension, and renal injury. The ALTITUDE study used the renin 
inhibitor alizarin in combination with ACEIs/ARBs in the treatment of high-risk cardio-cerebrovascular patients with type 2 diabetes complicated with hypertension and renal injury. The results showed that the combination treatment did not improve the cardiorenal endpoint events in the diabetic kidney disease patients, but it increased the incidence of adverse events. However, the patients included in these studies were generally older and had multiple risk factors. For example, the ALTITUDE trial included patients $>35$ years old, and the enrolled subjects were 65 years old on average. Therefore, these patients naturally belong to a high-risk group of cardiorenal endpoint events. However, this finding does not exclude the feasibility of combined therapy strategies in specific populations and specific disease stages. For example, young patients or patients without multiple cardiovascular risk factors may gain more benefits and have fewer adverse reactions using a dual RAS blockade strategy in the early stage of diabetic nephropathy.

A key problem associated with the dual RAS blockade strategy is adverse reactions. Currently, the most important safety problem related to the dual RAS blockade strategy is hypotension, which may lead to syncope and impaired renal function (indirectly leading to acute renal injury and hyperkalemia). The second-most important safety issue is hyperkalemia. Previous studies showed that the incidence of hyperkalemia (serum potassium $>5.5$ $\mathrm{mmol} / \mathrm{L}$ ) in hypertensive patients without risk factors for hyperkalemia treated with 1 RASis was $2 \%$, and the corresponding value in patients treated with a dual RAS blockade strategy was as high as $5 \%$. The incidence of hyperkalemia was also higher in patients with CKD or heart failure [27, 28]. Makani et al. [29] performed a meta-analysis of 33 RCTs with 68,405 patients and noted that dual RAS blockade did not have significant benefits for patients compared with 1 RASi and did not reduce cardiovascular mortality. However, the double RAS blockade therapy reduced the admission rate of patients with heart failure by approximately $18 \%$, increased the risk of hyperkalemia by approximately $55 \%$, increased the risk of low blood pressure by approximately $66 \%$, and increased the risk of renal failure by approximately $41 \%$. Although the conclusion of the above study argues against the use of dual RAS blockade, a subgroup analysis based on age and risk factors was not performed. Therefore, the feasibility of a dual RAS blockade strategy in specific populations cannot be simply denied. Nussberger and Bohlender [30] define "optimal" RAS blockade as the maximal blockade achieved without causing hypotension, hyperkalemia, or renal dysfunction. Therefore, individualized dual RAS blockade may be considered an "optimal" RAS blockade strategy. The possible safety problems caused by the dual RAS blockade strategy may be improved using various measures, such as education for patients, families, and doctors (e.g., familiarity with the sources of dietary or supplemental potassium and measures to reduce potassium intake), the use of potassium binders, such as sodium zirconium cyclic silicate powder, to reduce the risk of complications, and more accurate screening for suitable patients, which involves 2 parameters. The first parameter involves screening patients who are more likely to benefit from the treatment, and the second parameter involves screening patients who are not prone to adverse reactions $[27,31,32]$. Therefore, young people who are in the early stage of cardiorenal diseases, such as hypertension and diabetes, with fewer risk factors, good compliance, and a high potential benefit-to-risk ratio, may be the "ideal" group to use the dual RAS blockade strategy. Therefore, we can consider selecting drugs with superior pharmacological characteristics and designing large sample prospective RCTs to evaluate the long-term effects of dual RAS blockade strategy in this population. Due to the theoretically lower incidence of adverse reactions in these patient populations, longer follow-up time may be required to assess the impact on the prognosis of cardiorenal endpoint events. Therefore, patients with better compliance must be included in this study design, and an efficient chronic disease management team should be established for the follow-up management of patients. Proteinuria remission rate and blood pressure control rate may also be used as evaluation indexes of this study to evaluate the efficacy of dual RAS blocking strategy more comprehensively. If the benefits from cardiorenal endpoint events are demonstrated in this "ideal" group, then we have more reasons to examine and optimize the use of dual RAS blockade strategy in high-risk groups.

Nephrologists want to try dual RAS blockade to reduce proteinuria in proteinuria nephropathy and improve the renal prognosis of CKD patients [33]. Early CKD patients tolerate RASis better when there is no multitarget organ damage. Therefore, nephrologists are more likely to use a dual RAS blockade strategy than cardiologists, especially in patients with proteinuria nephropathies who are not suitable for immediate use of immunosuppressants, such as IgA nephropathy [34]. Personalized medication and observation of adverse reactions are very important in these patients. Ruggenenti et al. [35] used individualized double RAS blockade for the treatment of nondiabetic nephropathy and found that it more effectively reduced proteinuria and slowed the progression of nephropathy 


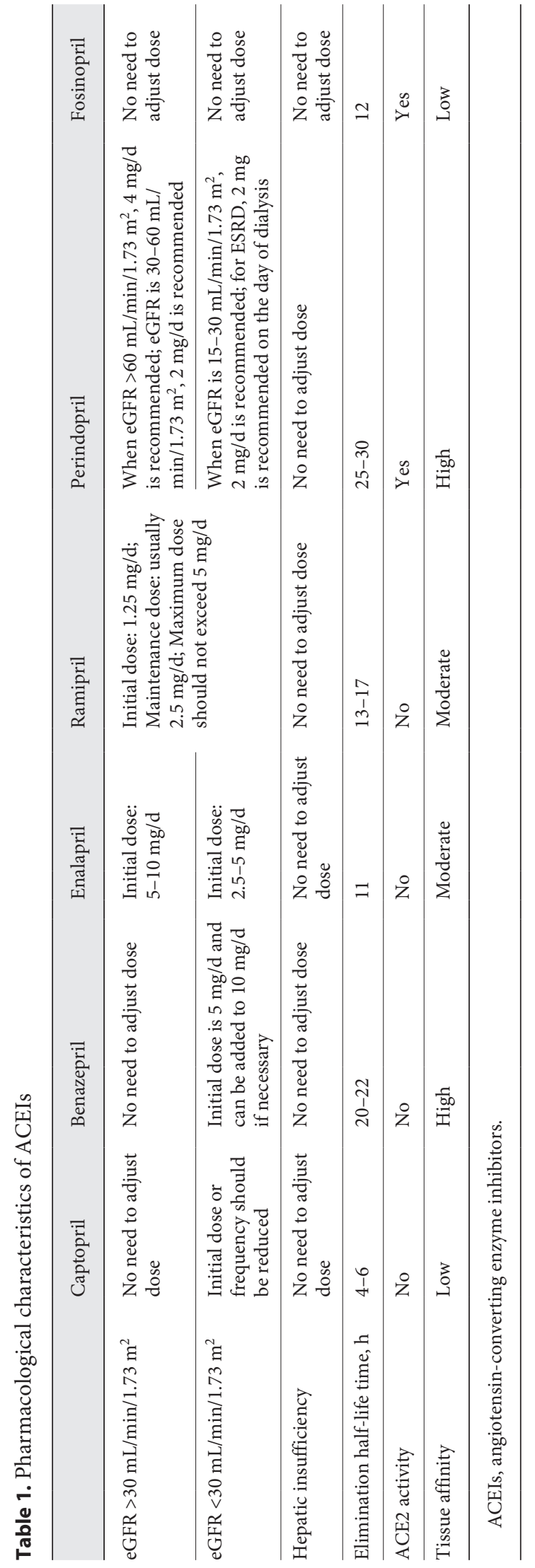

Dual Blockade Strategy for Cardiorenal Diseases

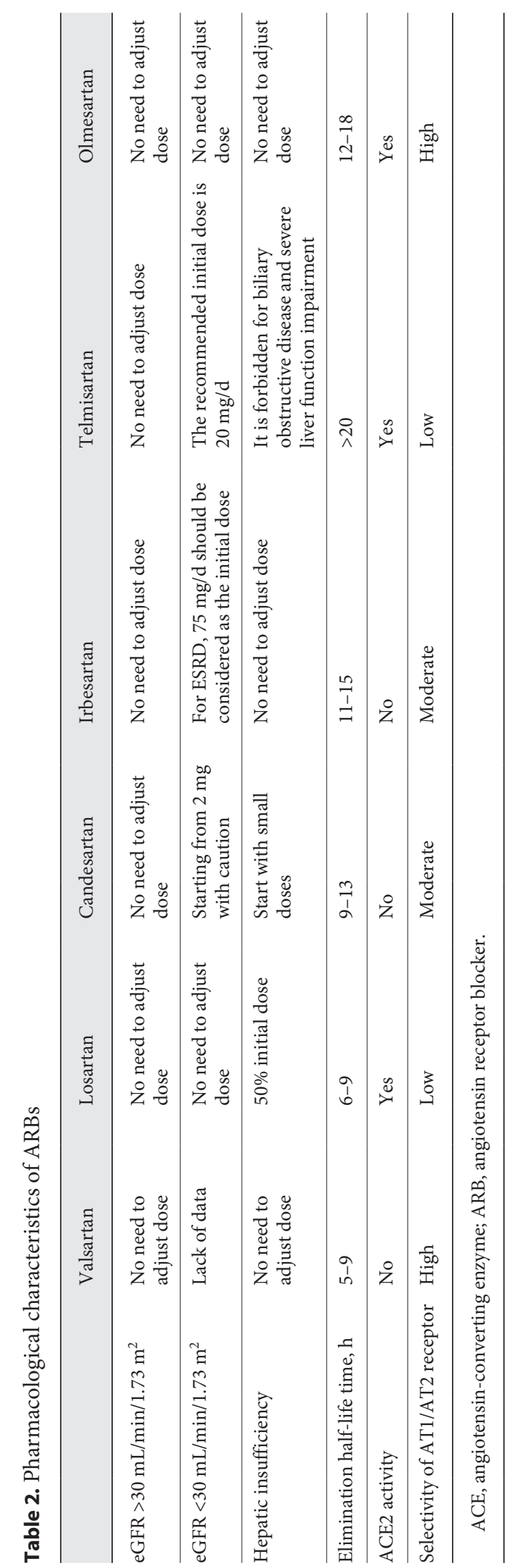

Nephron 2021;145:99-106 
than single RAS blockade. Many subsequent meta-analyses confirmed the hypothesis that dual RAS blockade was more effective in regulating albuminuria than single RAS blockade. For patients to obtain additional benefits, it is necessary to choose individualized treatments $[34,36]$. However, for possible safety reasons, such as high potassium and adverse reactions of renal function injury, the VA NEPHRON-D study was stopped prematurely, which led many nephrologists to abandon the dual RAS blockade strategy.

However, the combining of RASis for multitarget therapy may improve the efficiency of RAS blockade and the antiproteinuric effect and reduce the incidence of adverse reactions while retaining their unique renal protection mechanisms in theory. A review published in Nature Reviews Nephrology titled "Welcome Back" clearly proposed reconsideration of the dual blockade strategy for RAS [37]. The CALM study reviewed previous clinical studies and reported the benefits of dual blockade of RAS in humans for the first time. Although early clinical studies showed a higher incidence of adverse reactions, these studies included older groups, and most patients had cardiovascular complications, which placed these patients in high-risk groups for the use of RASis. For patients who are younger with no cardiovascular complications, a systematic evaluation is lacking [38]. Esteras et al. [39] systematically evaluated the risks of hyperkalemia, hypotension, and impaired renal function with the combination of 2 types of RASis and summarized that the door was left open to dual blockade therapy individualization although renal function, electrolytes, and blood pressure should be closely monitored [39]. Another study investigating a combination of RASi drugs from German sanatoriums derived the same conclusion: the risks and benefits of dual RAS blockade must be evaluated on an individual level, and strict safety inspection and subsequent regulatory actions are key to ensure the safe use of drugs [40]. These studies show that the adverse reactions may be effectively avoided via better patient education and prescribing skills, and should not be used as a reason to simply deny dual blockade strategy.

In the latest systematic review and meta-analysis of dual RAS blockade for the treatment of heart failure and renal insufficiency, 34,131 patients were selected from 12 of 2,258 articles. The hospitalization and adverse events, including all-cause death, heart failure, and cardiovascular death, in patients receiving dual RAS blockade, were investigated. Compared to monotherapy, dual RAS blockade reduced the death risk ratio of all individuals, significantly reduced the hospitalization rate of heart failure and cardiovascular death, reduced the hospitalization rate of heart failure and cardiovascular death in patients with renal disease, and reduced the cardiovascular hospitalization rate, hyperkalemia, and hypotension. These results suggest that dual RAS blockade reduced the risk of cardiovascular death and hospitalization for heart failure but did not increase the risk of renal damage. However, specific adverse events must be monitored during treatment [41]. In a RCT, Saglimbene et al. [42] reported that patients may tolerate ARBs better than ACEIs. However, the data reported in this article showed no differences in mortality or renal outcomes with ACEIs or ARBs as dual or monotherapy in patients with albuminuria and other cardiovascular risk factors.

\section{Individualized Drug Selection According to Pharmacological Characteristics}

Instead of debating whether ACEIs or ARBs are the better drug, clinicians should focus on understanding the pharmacological characteristics of each drug and provide individualized medication. The physicochemical properties and pharmacological characteristics of RASis, including the drug half-life time, antihypertensive ability, tissue affinity, metabolic pathway, and interactions with other drugs, are quite different. Only by understanding their basic characteristics can we make individualized and accurate drug choices for patients. For example, given their pharmacological characteristics, the early developed drugs captopril and fosinopril should not be used for dual RAS blockade because of their low-tissue affinity, RAS escape, and inability to increase ACE2 activity. Subsequently developed drugs, such as perindopril, overcome the aforementioned shortcomings and are good drug candidates for dual RAS blockade because of the reduced probability of adverse reactions. For ARBs, we can focus on the selectivity of AT1/AT2 receptors, half-life time, metabolic pathways, and compatibility with ACEIs. Only via a reasonable combination of ACEIs and ARBs can we maximize their strengths, avoid weaknesses, and achieve the purpose of dual RAS blockade. We provide a summary of the characteristics of clinical ACEIs and ARBs in Tables 1 and 2. After weighing their pharmacological characteristics, we think that perindopril/olmesartan may be a better option for future RCT study of dual RAS blockade strategy. 


\section{Conclusion}

The optimal RAS blockade should be defined by the maximum blocking effect that does not cause adverse reactions, such as hyperkalemia, hypotension, or renal failure. Therefore, it is time to reconsider the strategy of combination therapy because this strategy may be an effective tool to safely reduce or prevent the progression of cardiorenal diseases and provide individualized dosing and tolerance. The abandonment of this plan because of adverse reactions that may be avoided via technical means is logically similar to stop eating for fear of choking. The other good news is that the newly developed RASis have superior pharmacological characteristics, such as better tissue permeability, a better capacity to regulate ACE2, and a better trough-to-peak ratio and half-life time, which are likely to compensate for the shortcomings of earlier drugs to a large extent. Therefore, the use of newly developed RASi drugs in new head-to-head RCTs to reevaluate the dual RAS blockade strategy is very important. If we can achieve individualized treatment via careful dose adjustment and the reasonable selection of patients, combined treatment with RASis could become an effective tool for cardiorenal protection.

\section{Acknowledgements}

We thank our colleagues at the Department of Nephrology, the First Hospital Affiliated to Army Medical University for their help in the formation of this article.

\section{Conflict of Interest Statement}

The authors have no conflicts of interest to declare.

\section{Funding Sources}

This study was supported by the Chongqing Science and health joint medical research general program (2018MSXM123).

\section{Author Contributions}

Mei Mei, Zulian Zhou, Qian Zhang, Yi Chen, Hongwen Zhao, and Bingbing Shen reviewed the literature and wrote the manuscript.

\section{References}

1 Mogensen CE, Neldam S, Tikkanen I, Oren S, Viskoper R, Watts RW, et al. Randomised controlled trial of dual blockade of renin-angiotensin system in patients with hypertension, microalbuminuria, and non-insulin dependent diabetes: the candesartan and lisinopril microalbuminuria (CALM) study. BMJ. 2000 Dec 9;321(7274):1440-4.

2 Cao Z, Cooper ME, Wu LL, Cox AJ, JandeleitDahm K, Kelly DJ, et al. Blockade of the reninangiotensin and endothelin systems on progressive renal injury. Hypertension. 2000 Oct; 36(4):561-8.

3 De Z, Remuzzi G, Parving HH. Albuminuria, a therapeutic target for cardiovascular protection in type 2 diabetic patients with nephropathy. Circulation. 2004;110:921-7.

4 http: //www.ema.europa.eu/docs/en_GB/ document_library/Press_release/2012/02/ WC500122913.pdf.

5 Yusuf S, Yusuf S, Teo KK, Pogue J, Dyal L, Copland I, et al. Telmisartan, ramipril, or both in patients at high risk for vascular events. N Engl J Med. 2008;358(15):1547-59.

6 Fried LF, Emanuele N, Zhang JH, Brophy M, Conner TA, Duckworth W, et al. Combined angiotensin inhibition for the treatment of diabetic nephropathy. N Engl J Med. 2013; 369(20):1892-903.
7 Parving $\mathrm{HH}$, Brenner BM, McMurray JJ, de Zeeuw D, Haffner SM, Solomon SD, et al. (2012) ALTITUDE investigators. Cardiorenal end points in a trial of aliskiren for type 2 diabetes. N Engl J Med. 2012 Dec 6;367(23): 2204-13.

8 Grolla E, Bonanni L, Cutolo A, Presotto F, Dalla Vestra M. Disputes in the treatment of diabetic nephropathy: the dual blockade of renin-angiotensin system. Exp Clin Endocrinol Diabetes. 2016;124(6):361-6.

9 Forrester SJ, Booz GW, Sigmund CD, Coffman TM, Kawai T, Rizzo V, et al. Angiotensin II signal transduction: an update on mechanisms of physiology and pathophysiology. Physiol Rev. 2018 Jul 1;98(3):1627-738.

10 Ames MK, Atkins CE, Pitt B. The renin-angiotensin-aldosterone system and its suppression. J Vet Intern Med. 2019 Mar-Apr;33(2): 363-82.

11 Santos RAS, Sampaio WO, Alzamora AC, Motta-Santos D, Alenina N, Bader M, et al. The ACE2/angiotensin-(1-7)/MAS axis of the renin-angiotensin system: focus on angiotensin-(1-7). Physiol Rev. 2018 Jan 1;98(1): 505-53.
12 Stoll D, Yokota R, Sanches Aragão D, Casarini $\mathrm{DE}$. Both aldosterone and spironolactone can modulate the intracellular ACE/ANG II/ AT1 and ACE2/ANG (1-7)/MAS receptor axes in human mesangial cells. Physiol Rep. 2019 Jun; 7(11):e14105.

13 Smyth LJ, Cañadas-Garre M, Cappa RC Maxwell AP, McKnight AJ. Genetic associations between genes in the renin-angiotensinaldosterone system and renal disease: a systematic review and meta-analysis. BMJ Open. 2019;9(4):e026777.

14 Arendse LB, Danser AHJ, Poglitsch M, Touyz RM, Burnett JC, Llorens-Cortes C, et al. Novel therapeutic approaches targeting the reninangiotensin system and associated peptides in hypertension and heart failure. Pharmacol Rev. 2019 Oct;71(4):539-70.

15 Kai H, Kai M. Interactions of coronaviruses with ACE2, angiotensin II, and RAS inhibitors-lessons from available evidence and insights into COVID-19. Hypertens Res. 2020 Apr 27;43(7):648-54.

16 Chapman A, Gunning S. Real-world associations between renin-angiotensin-aldosterone system blockade therapy, hyperkalemia, and outcomes: a clinical and scientific call to action. J Am Heart Assoc. 2019 Nov 19;8(22): e014845. 
17 Menard J, Campbell DJ, Azizi M, Gonzales MF. Synergistic effects of ACE inhibition and Ang II antagonism on blood pressure, cardiac weight and renin in spontaneously hypertensive rats. Circulation. 1997;96:3072-8.

18 Thangaraju P, Chakrabarti A, Banerjee D, Hota D, Tamilselvan, Bhatia A, et al. Dual blockade of renin angiotensin system in reducing the early changes of diabetic retinopathy and nephropathy in a diabetic rat model. N Am J Med Sci. 2014 Dec;6(12):625-32.

19 Cherney DZI, Scholey JW, Jiang S, Har R, Lai $\mathrm{V}$, Sochett EB, et al. The effect of direct renin inhibition alone and in combination with ACE inhibition on endothelial function, arterial stiffness, and renal function in Type $1 \mathrm{di}-$ abetes. Diabetes Care. 2012 Nov;35(11): 2324-30.

20 Nakao N, Yoshimura A, Morita H, Takada M, Kayano T, Ideura T, et al. Comination treatment of angiotensin II receptor blocker and angiotensin-converting-enzyme inhibitor in non-diabetic renal disease (COOPERATE):a randomized controlled trial. Lancet. 2003; 361:117-24.

21 Weir RA, McMurray JJ, Puu M, Solomon SD, Olofsson B, Granger CB, et al. Efficacy and tolerability of adding an angiotensin receptor blocker in patients with heart failure already receiving an angiotensin-converting inhibitor plus aldosterone antagonist, with or without a beta blocker. Findings from the candesartan in heart failure: assessment of reduction in mortality and morbidity (CHARM)-added trial. Eur J Heart Fail. 2008 Feb;10(2):157-63.

22 Krum H, Carson P, Farsang C, Maggioni AP, Glazer RD, Aknay N, et al. Effect of valsartan added to background ACE inhibitor therapy in patients with heart failure: results from Val-HeFT. Eur J Heart Fail. 2004 Dec;6(7): 937-45.

23 Whelton PK, Carey RM, Aronow WS, Casey JrDE, Collins KJ, Himmelfarb CD, et al. ACC/ $\mathrm{AHA} / \mathrm{AAPA} / \mathrm{ABC} / \mathrm{ACPM} / \mathrm{AGS} / \mathrm{APhA} /$ ASH/ASPC/NMA/PCNA guideline for the prevention, detection, evaluation, and management of high blood pressure in adults: a report of the American college of cardiology/ American heart association task force on clinical practice guidelines. J Am Coll Cardiol. 2017.
24 ESC (European Society of Cardiology) and ESH (European Society of Hypertension). 2018 ESC/ESH guidelines for themanagement of arterial hypertension. Eur Heart J. 2018:1-98.

25 Mallat SG. Dual renin-angiotensin system inhibition for prevention of renal and cardiovascular events: do the latest trials challenge existing evidence?. Cardiovasc Diabetol. 2013;12:108.

26 Weir MR, Bakris GL, Bushinsky DA, Mayo MR, Garza D, Stasiv Y, et al. Patiromer in patients with kidney disease and hyperkalemia receiving RAAS inhibitors. $\mathrm{N}$ Engl $\mathrm{J}$ Med. 2015 Jan 15;372(3):211-21.

27 Van Buren PN, Adams-Huet B, Nguyen M, Molina C, Toto RD. Potassium handling with dual renin-angiotensin system inhibition in diabetic nephropathy. Cjasn. 2014;9(2):295-301.

28 Ingelfinger $\mathrm{J}$. A new era for the treatment of hyperkalemia? N Engl J Med. 2015;372(3): 275-7.

29 Makani H, Bangalore S, Desouza K, Shah A, Messerli F. Efficacy and safety of dual blockade of the renin-angiotensin system: metaanalysis of randomised trials. BMJ. 2013;346: f360.

30 Nussberger J, Bohlender J. Optimal blockade of the renin-angiotensin-aldosterone system. Nat Rev Cardiol. 2013;10(4):183-4.

31 Caravaca-Fontán F, Valladares J, DíazCampillejo R, Barroso S, Luna E, Caravaca F. Detrimental effect of renin-angiotensin blockade on progression of chronic kidney disease at later stages: a matter of dosage adjustment? Nefrología. 2020 Jan-Feb;40(1): 38-45.

32 Chapman A, Gunning S. Real-world associations between renin-angiotensin-aldosterone system blockade therapy, hyperkalemia, and outcomes: a clinical and scientific call to action. J Am Heart Assoc. 2019 Nov 19;8(22): e014845.

33 Campbell R, Sangalli F, Perticucci E, Aros C, Viscarra C, Perna A, et al. Effects of combined ACE inhibitor and angiotensin II antagonist treatment in human chronic nephropathies. Kidney Int. 2003;63(3):1094-103.
34 Cheng J, Zhang X, Tian J, Li Q, Chen J. Combination therapy an ACE inhibitor and an angiotensin receptor blocker for IgA nephropathy: a meta-analysis. Int J Clin Pract. 2012; 66(10):917-23.

35 Ruggenenti P, Perticucci E, Cravedi P, Gambara V, Costantini M, Sharma SK, et al. Role of remission clinics in the longitudinal treatment of CKD. J Am Soc Nephrol. 2008;19(6): 1213-24.

36 Palmer SC, Mavridis D, Navarese E, Craig JC, Tonelli M, Salanti G, et al. Comparative efficacy and safety of blood pressure-lowering agents in adults with diabetes and kidney disease: a network meta-analysis. Lancet. 2015 May 23;385(9982):2047-56.

37 Ruggenenti P, Remuzzi G. RAS blockade: nephroprotection by dual RAS blockade - a welcome back. Nat Rev Nephrol. 2015 Sep; 11(9):507-8.

38 Jafar TH, Assam PN. Dual RAAS blockade for kidney failure: hope for the future. Lancet. 2015 May 23;385(9982):2018-20.

39 Esteras R, Perez-Gomez MV, Rodriguez-Osorio L, Ortiz A, Fernandez-Fernandez B. Combination use of medicines from two classes of renin-angiotensin system blocking agents: risk of hyperkalemia, hypotension, and impaired renal function. Ther Adv Drug Saf. 2015 Aug;6(4):166-76.

40 Dörks M, Herget-Rosenthal S, Hoffmann F, Jobski K. Combined use of drugs inhibiting the renin-angiotensin system: prescribing patterns and risk of acute kidney injury in German nursing home residents. Clin Interv Aging. 2018;13:1035-42.

41 Silva AR, Martini AG, Canto GL, Guerra ENDS, Neves FAR. Effects of dual blockade in heart failure and renal dysfunction: systematic review and meta-analysis. J Renin Angiotensin Aldosterone Syst. 2019 OctDec;20(4).

42 Saglimbene V, Palmer SC, Ruospo M, Natale $\mathrm{P}$, Maione A, Nicolucci A, et al. The long-term impact of renin-angiotensin system (RAS) inhibition on cardiorenal outcomes (LIRICO): a randomized, controlled trial. J Am Soc Nephrol. 2018 Dec;29(12):2890-9. 